\title{
Improving the recognition of post-operative acute kidney injury
}

\author{
Nicola Trotter, Cal Doherty, Vicki Tully, Peter Davey, Samira Bell
}

NHS Tayside, Scotland

\begin{abstract}
The National Institute for Health and Care Excellence (NICE) state that acute kidney injury (AKI) is seen in 13-18\% of all people being admitted to hospital and that other patients will further go on to develop AKI during their time in hospital, with around $30-40 \%$ being in the operative setting. AKI has an estimated inpatient mortality of $20-30 \%$ in the UK and can lead to long-term morbidities like chronic kidney disease.[2]
\end{abstract}

AKI is under-recognised and badly managed despite its prevalence and seriousness, with NCEPOD report stating that only $50 \%$ of patients with AKI received good care, that there was poor assessment of risk factors for AKI, and there was an unacceptable delay in recognising postadmission $\mathrm{AKI}$ in $43 \%$ of patients.[4]

Baseline data collected on the urology ward in Ninewells Hospital, showed that only five of 22 (23\%) patients undergoing urological surgery had post-operative creatinine measured on the ward within 48 hours (the primary method for detecting AKI). Excluding patients who were discharged the same day $5 / 16(31 \%)$ received the blood test.

The aim of the project was to increase the number of patients returning to ward 9 post-surgery who receive a serum creatinine measurement within two days of their urological surgery, excluding daycases. Specifically, we wanted the reliability of this measurement to be $95 \%$ or over in ward 9 by 30 July 2014 .

This was to be done by raising awareness around AKI on ward 9 and changing protocol so that every patient staying on ward 9 beyond their day of surgery should receive a post-operative creatinine. This would be tested for a set amount of time to see if patients with AKI were being missed.

Despite not being able to implement a set protocol, the percentage of patients receiving post-operative creatinine measurements on ward 9 after a urological surgery still increased significantly. By interacting with the urology team and presenting our data, the knowledge and comprehension of the problem was altered. This lead to a change in culture and a significant increase in the number of post-operative creatinine measurements being taken. Through building relationships on the ward and sharing our data and knowledge there was an increase from $27 \%$ of patients receiving post-op creatinine in our first week of collecting data, to $87 \%$ in our last week on ward 9 . However, without a set tool or change in protocol this change appears to have not been sustainable as the percentage dropped to $42 \%$ two weeks later.

\section{Problem}

Acute kidney injury (AKI) is a serious health condition and is associated with an increased hospital stay, higher risk of hospital mortality, and an increased risk of progression to chronic kidney disease (CKD).[1] Patients undergoing surgery can go on to develop AKI post-operatively, particularly if additional risk factors are present.[2]

Postoperative AKI can be detected, by comparing pre-operative and post-operative creatinine levels, as well as closely monitoring urine output.[2] An early and effective management plan can then be implemented to improve recovery and reduce short and long term complications.[2]

A study in the Tayside area, supported by Scottish Government's HAI Task Force, investigated the possibility that changes in gentamicin prescription had the unintended consequence of increasing postoperative acute kidney injury (AKI).[3] The study unveiled that a significant proportion of patients were developing $\mathrm{AKI} ;>10 \%$ of patients undergoing gastrointestinal, orthopaedic, vascular, and urology procedures. It also found that patients were developing AKI after their operation, regardless of gentamicin prescription, and that this was not identified due to gaps in postoperative creatinine measurement. One observation of this study was that only around $55 \%(n=360)$ of patients undergoing urological surgery received a post-operative creatinine measurement. This is seen to be unacceptable by the renal team who say that the majority of these patients should receive a routine post-operative creatinine measurement as their urological surgery puts them at risk of developing AKI. The majority of these patients will receive prophylactic gentamicin, may be fluid restricted and may have prolonged urinary obstruction further increasing their risk of developing AKI.

\section{Background}

A 2009 report by the UK National Confidential Enquiry into Patient 
Outcome and Death (NCEPOD) reported that only $50 \%$ of patients with $\mathrm{AKI}$ received good medical care, that there was poor assessment of risk factors for $\mathrm{AKI}$, and there was an unacceptable delay in recognising post-admission $\mathrm{AKI}$ in $43 \%$ of patients.

In light of this report, the National Institute for Health and Care Excellence (NICE) produced guidelines on AKI in 2013. They stated that $A K I$ is seen in $13-18 \%$ of all people being admitted to hospital and other patients will go on to develop AKI during their time in hospital, with around $30-40 \%$ being in the operative setting.

Despite this welcome increase in research and awareness of AKI, knowledge and understanding on wards still seems to be suboptimal and this may be leading to inadequate recognition and treatment of AKI. Quality improvement projects published via BMJ Quality Improvement Reports, namely 'Acute Kidney Injury: It's as easy as ABCDE', and 'Improving the management of Acute Kidney Injury in a District General Hospital: Introduction of the DONUT bundle', both demonstrate and promote the effectiveness of increased awareness and education of staff to improve the prevention, detection and management of AKI.[5,6]

Although this a summer project which will be working primarily with patients undergoing urological surgical procedures, it is part of a greater initiative undertaken at Ninewells Hospital and nationally to improve care around $\mathrm{AKI}$, including improvements to the E-Alert system and the development of Tayside AKI guidelines.

\section{Baseline measurement}

Measures in the pre-operative and post-operative setting were considered. The first measure was the number of patients, who were undergoing urological surgical procedures, who had had their preoperative creatinine checked within three months of their surgery. Three months is considered valid, when comparing with post-operative creatinine.[2]

The second measure was the number of patients that were undergoing urological surgical procedures who had had their postoperative creatinine measured within 48 hours, as per the recommended time frame.[2] This second measure was divided into patients who returned to ward 9 (urology ward) and remained in hospital for only their day of surgery, one day after their day of surgery, and two or more days after their day of surgery. Patients who returned to the day surgery unit or to other wards were also included in these measures.

These measures were collected by using the online collecting and reporting system ICE and clinical portal. The baseline data was collected for one week. Twenty-four patients were scheduled to have urological surgery during this week. Two patients surgeries were postponed, and data was collected from the remaining 22 .

All bar two patients received a pre-operative creatinine measurement. Sixteen of $20(80 \%)$ patients had their pre-operative creatinine measured within three months of their operation.

Post-operatively only $5 / 22(23 \%)$ had their creatinine measured within 48 hours. Of the $15 / 22$ patients who returned to ward 9 only $4 / 15(27 \%)$ patients had post-operative creatinine measured on the ward within 48 hours. All patients that received the blood test stayed two or more days after their surgery. However, still only $4 / 8$ $(50 \%)$ of patients who stayed more than two days received the blood test. Zero out of seven patients that were discharged the same day $(n=4)$ or the following day $(n=3)$ had their serum creatinine measured. Two additional patients received the blood test within seven days, however this was in primary care and was not related to the urological surgery.

This data indicated that there may be problems in both the preoperative and postoperative systems. Furthermore these shortfalls may be leading to reduced recognition of AKI.

See supplementary file: ds4337.pptx - "Attachment 1- Baseline Data and Process Maps."

\section{Design}

The aim of our intervention is to increase the number of patients returning to ward 9 for over one day post-surgery who receive a serum creatinine measurement within two days of their urological surgery. Specifically, we want the reliability of this measurement to be $95 \%$ or over.

The first intervention was to engage with key stakeholders and staff to explain the project and increase their awareness of the problem. This would be conducted through arranging meetings with key staff members and talking to staff on the ward. The aim of this would be to gain approval and cooperation to implement a change in protocol and ensure that a change is sustainable.

A change in protocol on the ward would then be implemented so that all patients who stay beyond their day of surgery receive a serum creatinine measurement. This would be implemented for a set amount of time (four to six weeks) as a test for change, to observe whether there was an increase in creatinine measurements and an increase in recognition of AKI.

Possible changes to phlebotomy and to ward 9's discharge system would also be considered; this would be to ensure that patient's discharge was not unnecessarily delayed and to minimise the potential strain that this change could have on the system as a whole. These include:

1. Improving the use of the pneumatic tube pipelines present on ward 9 that can transport vacutainers straight to blood sciences for analysis by increasing the number of capsules available to ward 9. Presently, vacutainers are sent to blood sciences once the phlebotomist has finished the run, delaying when blood results can be returned

2. Revising the run order the phlebotomy service take when moving from ward to ward. Presently the run order starts in ward 7 and then 8,9 , and 10 . Ward 7 is the acute surgical receiving ward and would have to remain first, however if ward 9 were to follow this would mean blood would be obtained sooner 
3. Changing the discharge protocol on ward 9 whereby patients who are expected to be discharged that day remain in hospital until blood results return.

A management plan is set for the patient if $\mathrm{AKI}$ is found, depending on the stage. They are still discharged if at stage 1 and the patient is fit, and receive a repeat blood test in primary care. If stage 2 or 3 the patient must remain in hospital for further observation and the renal team should be notified (process map 5).

Data collection will be continued to assess whether there has been any improvement and to increase our evidence base in each category of patients.

\section{Strategy}

The first PDSA cycle's aim was to collect data surrounding patients undergoing urological surgery for one week and develop a tool to easily collect the data. This data will show how many patients receive a post-operative creatinine after their urological operation and when this blood test occurs. The process for collecting data was improved and the amount of time needed to collect a weeks worth of data was reduced, mainly through the use of online systems like ICE and clinical portal (process map 6). The predicted conclusion that not enough patients were getting their postoperative creatinine measured was proven through the data collected. This allowed us to begin creating possible interventions to improve this.

The second PDSA cycle's aim was to raise awareness of the problem, identified by the data collected among key stakeholders and members of staff. This included increasing knowledge of AKI on the ward and gaining approval and cooperation to implement a change in protocol. Inclusion of enthusiastic members of staff who could play an active role in the project was an additional aim. This was achieved by arranging meetings with key individuals who were able to implement a change in protocol and spread awareness throughout their team.

Engaging and raising awareness among staff on ward 9 was successful, particularly among junior doctors and specific nurses, because we were present on ward 9 and so working relationships developed. However, we endured a great deal of resistance when we tried to engage other key stakeholders, such as the urological consultants. They put in place several barriers such as requesting ethical consent and it was challenging to arrange a meeting with them.

However, a stark increase in the number of postoperative creatinine measurements occurred which we believe was due to the Hawthorne Effect. Our presence on ward 9 and the awareness raised seemed to result in staff trying to improve the system themselves and hence a much greater improvement occurred than expected. There was an increase of postoperative creatinine measurements, from $38 \%$ in the baseline to an average of $98 \%$ over the four week period in patients who stayed two or more days beyond their day of surgery. Patients who were discharged the day after their day of surgery also showed an improvement; from $0 \%$ to an average of $40 \%$.

In order to ensure this change was sustainable, our next PDSA cycle's plan was to change the protocol surrounding post-operative creatinine measurements in patients undergoing urological surgical procedures who stay in hospital for more than one day after their day of surgery. Despite the approval and agreement from key stakeholders for the intervention, further delays in the organisation of multidisciplinary meetings meant there was no time left to implement a formal change as backing from key stakeholders was necessary.

Therefore, the original change in postoperative creatinine observed began to decrease as our presence on ward 9 decreased. There was also a change in junior doctor staffing which would also explain this reduction. However, by returning to the ward and explaining our project to new staff, the percentage of patients who stayed over two days post-operative and who received a post-operative creatinine, rose to $100 \%$ again. We hope by continuing to meet with key stakeholders and by passing the project on to other members of the team that we have engaged with, the percentage of patients receiving a postoperative creatinine will once again rise.

See supplementary file: ds4338.docx - "Attachment 2- PDSA Cycle Summary."

\section{Post-measurement}

Seven weeks of data was collected, including the first week's baseline measurement. The data collected was the number of patients that were undergoing urological surgical procedures who had had their post-operative creatinine measured within 48 hours of surgery. It was collected using the data collection tool devised, using online clinical systems like ICE and clinical portal.

This was then analysed. Firstly all together (run chart 1) excluding day-case patients, then split into patients who were discharged the day after their surgery (run chart 2) and patients who stayed two or more days after their surgery (run chart 3). Due to fluctuating patient numbers per day, this data was analysed per week.

The data was then organised into run charts and changes were noted and improvements or regressions were explained.

See supplementary file: ds4340.docx - "Attachment 3- Runcharts"

\section{Lessons and limitations}

A multidisciplinary team approach to improving the number of patients who receive a post-operative creatinine may have improved the success of the project. We formed a strong relationship with the staff on the urology ward but did not spend a lot of time engaging with other stakeholders. In hindsight, engaging all the stakeholders at the beginning may have reduced several barriers we faced. Instead of focusing simply on the team on the ward that we were in contact with on a daily basis, it is important to identify the full team and engage all members early on in the project 


\section{BMJ Quality Improvement Reports}

and organise multidisciplinary team meetings and discussions so everyone is in agreement.

Moreover, tackling a large project and then trying to scale the problem down in order to focus on a specific area is challenging as it is difficult to know which route to follow and there is a sense of feeling that you should be tackling all areas needing improved (ie pre-assessment clinic, day surgery unit, ward 9). Therefore, understanding the problem and defining a focused goal at the beginning of the project would be beneficial.

\section{Conclusion}

There are many factors that contribute to the low percentage of patients receiving post-operative creatinine measurements. By interacting with the urology team and presenting our data, the knowledge and comprehension of the problem was altered. This lead to a change in culture and a significant increase in the number of post-operative creatinine measurements being taken. However, without a set tool or change in protocol it is unclear whether this change will be sustained. It is difficult to know whether the provision of real-time feedback and constant monitoring may be the main driver of this change.

\section{References}

1. Lewington A, Kanagasundarum S; UK Renal Association. UK Renal Association Clinical Practice Guideline on Acute Kidney Injury (5th edition). 2011. Available from: www.renal.org

2. Acute kidney injury - Prevention, detection and management of acute kidney injury up to the point of renal replacement therapy; NICE Guidelines. 2013. Available from: www.nice.org.uk/guidance

3. Bell S, Davey P, Nathwani D, Marwick C, Vadiveloo T, Sneddon $\mathrm{J}$ et al. Risk of AKI with gentamicin as surgical prophylaxis. J Am Soc Nephrol 2014 Nov;25(11):2625-32. Available from: www.jasn.asnjournals.org

4. National confidential Enquiry into Patient Outcome and Death (NCEPOD). Adding insult to Injury. A review of the care of patients who died in hospital with a primary diagnosis of acute kidney injury (acute renal failure). 2009. Available from: www.ncepod.org

5. Forde C, McCaughan J, Leonard N. Acute Kidney Injury: It's as easy as ABCDE. BMJ Quality Improvement Reports. 2013.

6. Bhagwanani A, Carpenter R, Yusuf A. Improving the management of acute kidney injury in a district general hospital. Introduction of the DONUT bundle. BMJ Quality Improvement Reports. 2014.

\section{Declaration of interests}

NHS Tayside Added Costs of Teaching (ACT) funding each for a six week vacational scholarship.

\section{Acknowledgements}

Mr Andrew Martindale, Ms Morag MacRae. 